\title{
Bleiben Sie dem Nichtrauchen treu!
}

$\mathrm{V}$ on der Greifswalder Tagung im vergangenen Herbst meldet sich in dieser Ausgabe des Allergo Journal die Epidemiologie mit drei interessanten und aktuellen Beiträgen, die ich Ihnen gerne zur Lektüre empfehlen darf.

Aktuell ist das Thema Rauchen und Nichtraucherschutz. Am 23. Februar haben sich die Gesundheitsminister von Bund und Ländern grundsätzlich auf ein Rauchverbot in Gaststätten geeinigt. Danach dürfen Raucher nur noch in abgetrennten und gekennzeichneten Räumen zur Zigarette greifen, wobei die Bundesländer Nordrhein-Westfalen und Niedersachsen noch über Ausnahmen nachdenken. Dieser Schritt ist für Deutschland längst überfällig, liegen wir doch im europäischen Vergleich bezüglich des Nichtraucherschutzes deutlich zurück. $\mathrm{Ob}$ die Beschlüsse der Bund-Länder-Arbeitsgruppe die Grundlage für die Entscheidung der Ministerpräsidentenkonferenz legen konnten, werden wir am 22. März wissen.

Vielbelegte Tatsache ist, dass die Passivrauchexposition das Risiko für respiratorische atopische Erkrankungen, aber auch für das atopische Ekzem signifikant erhöht. In den aktuellen Daten der
„Ein Rauchverbot in Gaststätten ist für Deutschland längst überfällig, liegen wir doch im europäischen Vergleich bezüglich des zurück." Nichtraucherschutzes deutlich ISAAC-Studie, die von Herrn Genuneit vorgestellt werden (S. 103), wird gezeigt, dass Aktivrauchen bei Jugendlichen die Inzidenz des Asthmas signifikant erhöht. Für die Untersuchung in Dresden und München wurden die rund 3.000 initial 9- bis 11-Jährigen nach sieben Jahren noch einmal nachuntersucht.

Im zweiten Beitrag macht Herr Pohlabeln anhand der von ihm durchgeführten Geburtskohortenstudie deutlich, dass das Ernährungsverhalten, und hier insbesondere die Wahl hypoallergener Säuglingsnahrung, deutlich von der familiären Vorbelastung bezüglich allergischer Erkrankungen abhängt (S. 108).

Die Zahl der beobachteten Störche und die Geburtenrate in Deutschland haben beide abgenommen, ohne etwas miteinander zu tun zu haben. Zugenommen haben die

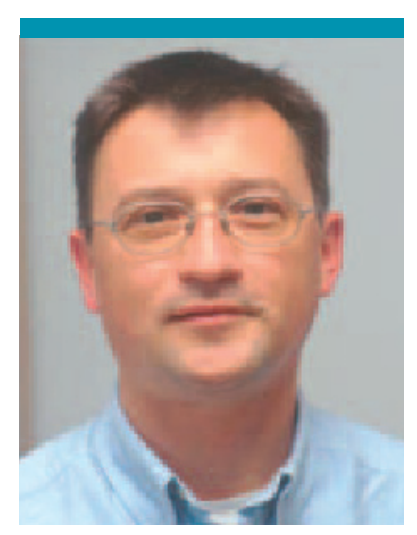

Prof. Dr. Torsten Schäfer, Institut für Sozialmedizin, Universitätsklinikum Schleswig-Holstein, Lübeck allergischen Erkrankungen und die Scheidungen. Während die Scheidungsquote zu Beginn des letzten Jahrhunderts deutlich unter 5\% lag, hat sie mit Beginn dieses Jahrhunderts die 50\%-Marke überschritten. Dass beide Phänomene etwas miteinander zu tun haben könnten, legen die Daten von Frau Bockelbrink nahe (S. 111).

Auf zwei Kongresse im September, auf denen Sie nicht nur, aber auch epidemiologische Beiträge hören werden, möchte ich hinweisen. Auf den Zweiten Gemeinsamen Deutschen Allergiekongress vom 26. bis 29. September in Lübeck (www.allergie-kongress. de) werden sie regelmäßig im Allergo Journal aufmerksam gemacht. Eine Woche zuvor findet in Augsburg vom 17. bis 21. September unter dem Motto „Prävention und Versorgung - innovativ - qualitätsgesichert - sozial“ der gemeinschaftliche Kongress Medizin und Gesellschaft zahlreicher Fachgesellschaften statt, die sich unter anderem mit den Themen Prävention und Epidemiologie beschäftigen (www.med-ges-2007.de). Vielleicht haben Sie ja Gelegenheit, an der einen oder anderen Veranstaltung teilzunehmen.

Ich wünsche Ihnen viel $S p a ß$ bei der Lektüre und bin mit herzlichen Grüßen

Ihr

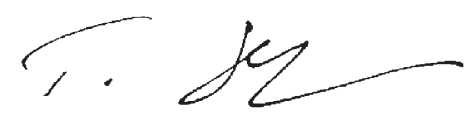

Prof. Dr. Torsten Schäfer, MPH 